\title{
Relaxation Lifetimes of Plasmonically Enhanced Hybrid Gold-Carbon Nanotubes Systems
}

M. Glaeske1,*, M. Kumar ${ }^{1}$, T. Bisswanger1， S. Vaitiekenas ${ }^{1}$, C. Soci ${ }^{2}$, R. Narula ${ }^{3}$, A. Bruno ${ }^{4,5}$,* and A. Setaro ${ }^{1, *}$.

${ }^{1}$ Department of Physics, Free University Berlin, Arnimallee 14, 14195 Berlin, Germany

${ }^{2}$ Division of Physics and Applied Physics, Nanyang Technological University, Singapore 637371

${ }^{3}$ Department of Physics, Indian Institute of Technology Delhi, Hauz Khas, New Delhi 110016, India

${ }^{4}$ Energy Research Institute at Nanyang Technological University (ERI@N), Research Techno Plaza, Singapore

${ }^{5}$ ENEA, Centro di ricerche di Portici, P.le Enrico Fermi, Portici (NA) Italy

*Email: mareen.glaeske@fu-berlin.de, Annalisa@ntu.edu.sg, setaro@physik.fu-berlin.de

\begin{abstract}
Recently, we introduced a novel hybridization route for carbon nanotubes using gold nanoparticles, whose close proximity neatly enhances their radiative emission. Here we investigate the mechanisms behind the enhancement by monitoring the de-excitation dynamics of our $\pi$-hybrids through two-color pump-probe time-resolved spectroscopy. The de-excitation process reveals a fast component and a slow component. We find that the presence of gold prominently affects the fast processes, indicating a stronger influence of the gold nanoparticle on the intra-band non-radiative relaxation than on the inter-band recombination of the SWNT. By evaluating the de-excitation times, we estimate the balance between near-field pumping and the faster metal-induced de-excitation contributions, proving the enhanced pumping to be the leading mechanism.
\end{abstract}




\section{Introduction}

Since the observation of their band gap photoluminescence (PL), semiconducting singlewalled carbon nanotubes (SWNTs) have emerged as promising candidates for applications in photonics, optoelectronics, nanoelectronics, and biological imaging [1]. Each nanotube species, uniquely identified by the chiral vector $\boldsymbol{c}=\left(n_{1}, n_{2}\right)$, can be seen as a specific material on its own, with its unique values of the excitation and emission energies [1], offering a broad set of materials for targeted applications. However, their low photoluminescence quantum yield, typically below one percent (and above only in exceptional cases) thwarts their widespread use in optical devices [2],[3]. To overcome this limit we introduced a new hybrid system to enhance their optical response. The $\pi$-hybrids, as we refer to them, are produced by swelling the micelles suspending gold nanorods (AuNRs) and encapsulating the SWNTs within. We termed them $\pi$-hybrids highlighting that the close proximity between the gold and the tubes affects primarily the $\pi$-bonds of the carbon network, which are known to dominate optical processes in SWNTs [4]. The presence of the metal boosts the emission of the hybrids -up to 20 times stronger than pristine tubes [5]. Other systems, such as SWNTs dropcasted on rough Au surfaces, have demonstrated enhanced SWNT emission as well [6]. In that work, it was concluded that the mechanism behind the enhancement for the tubes on the Au surface was the radiative lifetime shortening of the excited state. Here we demonstrate that in our colloidal systems, where every single SWNT is homogeneously exposed to the near-field from the metallic particles and not just the fraction randomly placed onto the surface plasmonic hotspot, this is not the dominant contribution to the enhancement process.

The interaction with different, more complex superstructures and their strong plasmonic modes, e.g. cavities based on plasmonic lattices obtained by e-beam lithography, have been shown to give rise to strong exciton-plasmon interactions and tunable Rabi-splitting of the cavity polaritonic modes [7],[8]. While highly promising for optoelectronic purposes, such 
top-down systems lack integrability within systems of biological relevance, where colloidal metallic nanoparticles have shown to be a better choice [9]. Here we focus on the effect of the interaction when an isolated metallic particle interacts with a nanotube.

One important parameter governing the phenomenology of their interaction is the emittermetal separation [6],[10]. Indeed, two different regions are formed around a nanometallic particle subjected to optical irradiation: the near- and the far-field region (highlighted as NF and FF in the sketch of Fig. 1a). In the near-field region (typically extending few nanometers around the metallic surface) the evanescent wave strongly squeezes in space along with an enhancement of the intensity of its electromagnetic field. In the far-field region we find the propagating component of the electromagnetic radiation scattered from the metallic particle. An emitter placed in the position labeled as (NF) in Fig. 1 will sense the plasmonic near-field, whereas an emitter placed in position (FF) will mostly sense the propagating component of the electromagnetic radiation, which can destructively or constructively interfere with the radiation generated by the emitter, yielding a net quenching or enhancement of the emitter's yield. These interference effects, though, occur for separations of the order of the metal particle radius or even bigger [10]. While they give rise to the enhancement of the SWNTs observed emission [11], they are negligible for the AuNR-SWNTs separations of our $\pi$ hybrids.

Generally, it is possible to depict the properties of the optical process through a simple 3-level system (cfr. Fig. 1a). The system at equilibrium is in its ground state. Upon excitation, it will populate the level that we label as $E_{22}$ for consistency with the standard nanotubes notation. From there, the system will undergo a fast nonradiative thermalization to the state labelled as $E_{11}$, from which it can de-excite both radiatively and nonradiatively. Please note that there could be additional states located between $\mathrm{E}_{11}$ and the ground state, causing additional deexcitation channels. They are taken in account by estimating the total lifetime of $\mathrm{E}_{11 .}$ 
(a)
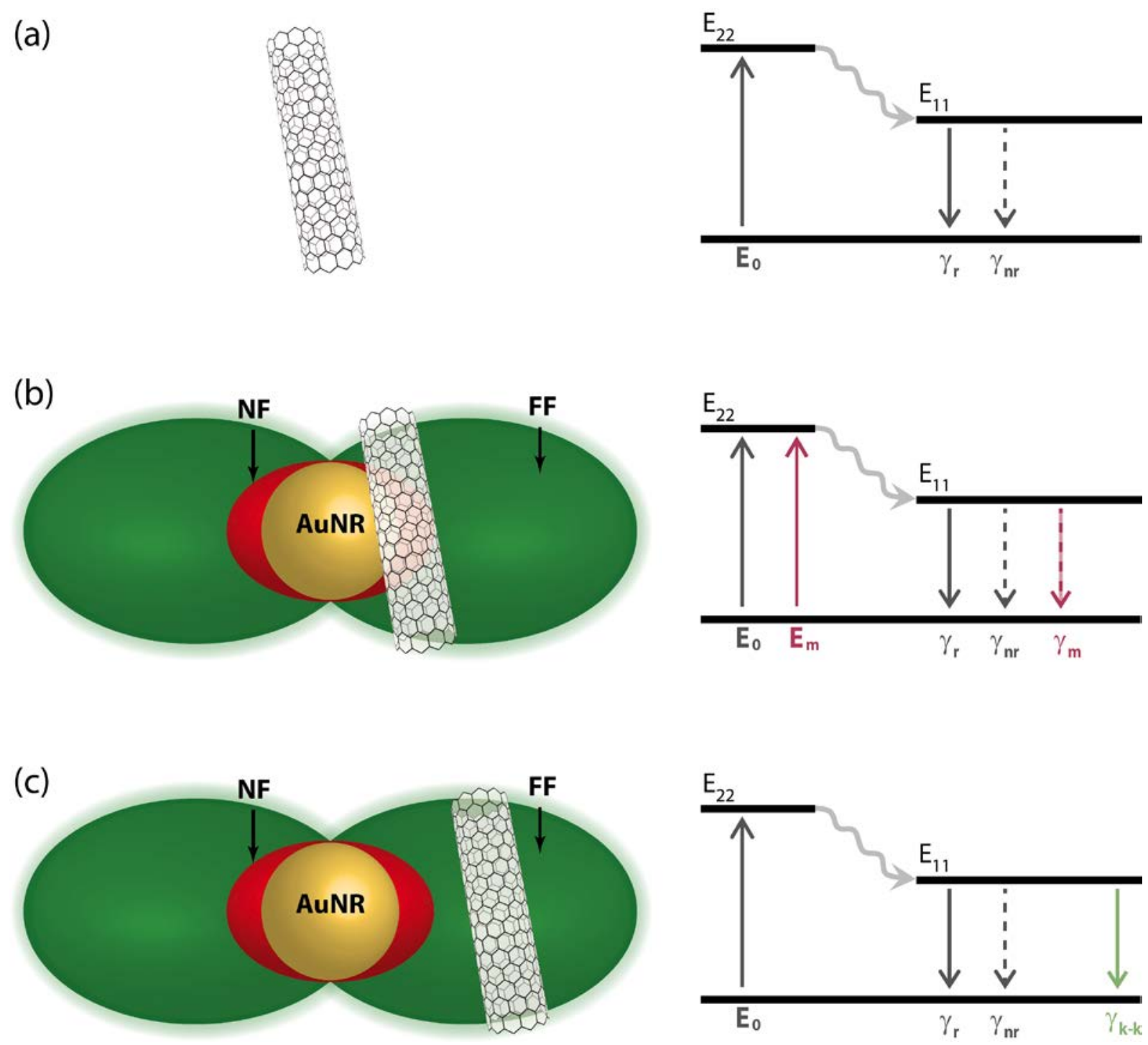

Figure 1 Schematic depiction of the distribution of the electromagnetic field around a small metallic nanoparticle (AuNR). The localized near-field is highlighted in red while the scattered, propagating component is green. Beyond the intrinsic excitation $\left(\mathrm{E}_{0}\right)$ and de-excitation $\left(\gamma_{\mathrm{r}}\right.$ and $\left.\gamma_{\mathrm{nr}}\right)$ pathways (a), the AuNR introduces additional excitation $\left(E_{m}\right)$ and de-excitation pathways $\left(\gamma_{\mathrm{m}}\right)$ due to the near-field interaction $(\mathbf{b})$. Additional interferential terms $\left(\gamma_{\mathrm{k}-\mathrm{k}}\right)$ are due to the interaction with the far-field (c).

The balance between these processes can result in different phenomenologies, ranging from enhanced optical response to total quenching. For emitters with low quantum yield and under weak illumination, it’s been shown that the emission is maximally enhanced by placing the emitter in the close proximity of the metallic surface [12], while competing quenching effects plays no significant role [13]. In such a lossy regime the enhancement factor $\Phi$ ascribed to proximity effects is determined by the balance between two contributions: The stronger pumping $F_{\text {exc }}$ and the faster de-excitation $F_{\text {rad }}[6],[10],[11]$ : 


$$
\Phi=F_{\text {exc }} \cdot F_{\text {rad }}
$$

Figure 1b provides a simplified sketch of the metal-induced changes in the optical process. The first term $F_{\text {exc }}$ of Eq. 1 takes into account the stronger pumping due to the locally enhanced plasmonic field in the proximity of the metallic surface in the transition from the ground state to $\mathrm{E}_{22}$ of Fig. 1b:

$$
F_{\text {exc }}=\left|\frac{E_{m}(\pi)}{E_{0}}\right|^{2}
$$

whereas the second term $\mathrm{F}_{\text {rad }}$ takes into account the metal-induced change in the de-excitation lifetimes in the transition from $E_{11}$ to the ground state depicted in Fig. 1b [11]:

$$
F_{\text {rad }}=\frac{\tau_{m}}{\tau_{0}}
$$

where $\tau_{0}=1 /\left(\gamma_{\mathrm{r}}+\gamma_{\mathrm{nr}}\right)$ is the total lifetime of the emitter in the free space and $\tau_{m}=$ $1 /\left(\gamma_{\mathrm{r}}+\gamma_{\mathrm{nr}}+\gamma_{\mathrm{m}}\right)$ is the emitter lifetime in the presence of the metal. The black arrows in Fig. $1 \mathrm{~b}$ indicate the intrinsic processes of the free emitter: $E_{0}$ is the excitation rate while $\gamma_{\mathrm{r}}$ and $\gamma_{\mathrm{nr}}$ are, respectively, the radiative (continuous line) and non-radiative (dashed line) deexcitation rates. Once the emitter interacts with the metal, additional excitation $\left(E_{m}\right)$ and deexcitation $\left(\gamma_{\mathrm{m}}\right)$ pathways arise. Note that we neglect the term labeled as $\gamma_{\mathrm{k}-\mathrm{k}}$ in Fig. 1c, which accounts for the interference effects in the far-field region.

In our previous works [5],[14], we had concluded that the key to efficient enhancement was the tube-gold proximity. To gain a deeper insight and identify which mechanism most efficiently contributes to the enhancement, here we account for the separate contributions from the stronger pumping and from the faster de-excitation occurring in Eq. 1. With this aim, we employ time resolved spectroscopy to monitor the de-excitation of our $\pi$-hybrids. 


\section{Experimental Details and Sample Preparations}

The SWNTs, produced by the CoMoCAT methods, were purchased from SWeNTs (SG 76). Hydrochloric acid (>99.5\% HCl, 37 wt. \% in water) was purchased from Carl Roth, and sodium oleate (NaOL, $>97.0 \%)$ from TCI. All other chemicals (Hexadecyltrimethylammonium bromide BioUltra, hydrogen tetrachloroaurate trihydrate $\left(\mathrm{HAuCl}_{4} \cdot 3 \mathrm{H}_{2} \mathrm{O},>99 \%\right)$, L-ascorbic Acid (>99\%), silver nitrate $\left(\mathrm{AgNO}_{3},>99 \%\right)$, sodium borohydride $\left.\left(\mathrm{NaBH}_{4}, 99 \%\right)\right)$ were purchased from Sigma Aldrich. The vessels were new and have been cleaned by sonication before use. We used ultrapure water (MilliQ). All the synthetic steps were carried out at $29^{\circ} \mathrm{C}$ in a water bath.

Our AuNRs were synthesized according to the method developed by Ye et al. using a CTABNaOL binary surfactant mixture. It is a seed-mediated two-step process, requiring first the formation of the Au nano-seeds and then their controlled growth into the AuNRs [15]. The seeds were synthesized by adding $1 \mathrm{~mL}$ of freshly prepared cold $\mathrm{NaBH}_{4}(6 \mathrm{mM})$ to a $10 \mathrm{~mL}$ $\mathrm{HAuCl}_{4}(0.25 \mathrm{mM})$ and CTAB $(0.1 \mathrm{M})$ solution. They were then left undisturbed for 30min before further use. To promote the growth of the seeds into rods, we added $0.48 \mathrm{~mL}$ of $\mathrm{AgNO}_{3}$ $(4 \mathrm{mM})$ solution to $10 \mathrm{~mL}$ of a CTAB-NaOL mixture, containing $8.1 \mathrm{mM} \mathrm{NaOL}$ and $47 \mathrm{mM}$ CTAB. After $15 \mathrm{~min}$ of stirring, we added $0.5 \mathrm{~mL} \mathrm{HAuCl}_{4}(10 \mathrm{mM})$ to trigger the prereduction phase. The solution was then gently stirred for $90 \mathrm{~min}$ at $29^{\circ} \mathrm{C}$. Next, we added $60 \mu \mathrm{L}$ of $\mathrm{HCl}$ (37\%) to adjust the $\mathrm{pH}$ value. After further stirring of $15 \mathrm{~min}$, we injected $25 \mu \mathrm{L}$ ascorbic acid and $8 \mu \mathrm{L}$ of the seed solution to start the growth process. The solution was left undisturbed for 12 hours.

We then prepared two different samples: The pristine tubes (without any gold, as a reference sample) and the $\pi$-hybrids. The samples were produced following the procedure detailed in our previous publications [5],[14]. The $\pi$-hybrids used in this work were produced by adding $210 \mu \mathrm{L}$ of uncoated CNTs solution $(0.1 \mathrm{~g} / \mathrm{L})$ to $1 \mathrm{~mL}$ of the gold suspension. The mixture was $-6 / 20-$ 
stirred for a week. It was left undisturbed for 3 days and the supernatant was used for the experimental characterization. The synthesis relies on the micelle-encapsulation technique, which requires adding uncoated SWNTs into a water sample containing AuNRs. The technique ensures that all the nanotubes introduced within the samples are in the close proximity of gold. In order to avoid tube-tube interaction and tube precipitation, we moreover work at low nanotubes concentration [5]. This ensures negligible nanotubes loss and similar chiral species distribution between the pristine and the hybrids samples (cfr. Figure 2c).

Continuous-wave excitation photoluminescence characterization of the samples was performed with a Nanolog spectrofluorometer from Horiba, equipped with a Xenon lamp and a liquid-nitrogen cooled InGaAs detector. The absorption spectra were acquired through a Perkin-Elmer Lambda 950 double-beam spectrophotometer with detector optics that can be compensated to take in account the samples optical density or thickness.

The time resolved measurements were carried out with a pump-probe setup, whose detailed description is given in Ref. [16]. We pumped our samples with the fundamental beam of a Ti:Sapphire femtosecond laser (wavelength $800 \mathrm{~nm}$, repetition rate $1 \mathrm{KHz}$, pulse width of around 100fs). The probe beam at $1100 \mathrm{~nm}$ was generated through an optical parametric amplifier. We selected the probe beam with a band-pass filter centered at $1100 \pm 2 \mathrm{~nm}$ and bandwidth of $50 \mathrm{~nm}$. The transient absorption signal was detected with a Thorlabs DET20C/M InGaAs biased detector connected to a Stanford Research Systems model SR830 DSP lock-in amplifier to select and amplify the probe signal at the pump chopping frequency. The time decays have been fitted with a double exponential decay curve signals deconvoluted with the instrumental response function (IRF, $100 \mathrm{fs}$ ). Indeed, any light signal we observe ( $\mathrm{I}_{\mathrm{obs}}$ ) is time convolution of the true profile ( $\left.\mathrm{I}_{\text {true }}\right)$ and the IRF. By knowing the latter, it is possible to retrieve the true profile through a common algorithm based on the method of Least Squares Iterative Reconvolution. This methodology has been proven to allow 
us to estimate decay times 10 shorter than the IRF itself. For further details on the technique, please refer to [17]-[19].

We kept the laser fluence as low as possible $\left(6 \times 10^{12}\right.$ photons $\mathrm{cm}^{-2}$ pulse $\left.^{-1}\right)$ to ensure detectable signals while avoiding complex multi-exciton phenomena [20].

\section{Results and Discussion}

\subsection{Morphology}

The optical and morphological properties of the plasmonic nanoparticles used in this work are depicted in Fig. 2a,b. Our gold nanoparticles exhibit transverse and longitudinal plasmon resonances, respectively, at $550 \mathrm{~nm}$ and $920 \mathrm{~nm}$. SEM analysis reveals a small polydispersion in the gold nanoparticles' morphology. The majority (approx. 85\%) of the particles are rods, $15 \mathrm{~nm}$ wide and $70 \mathrm{~nm}$ long. We will refer to them as AuNRs for the rest of this manuscript.

\subsection{Continuous-Wave Spectroscopy}
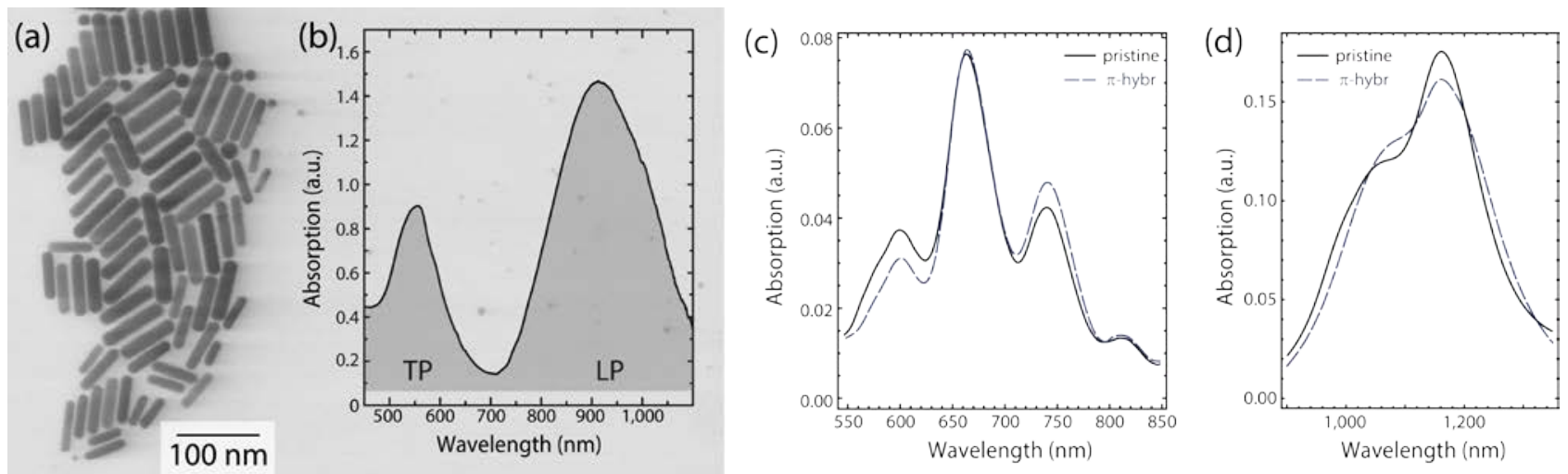

Figure 2 (a) SEM image of the used AuNRs. The sample is mostly composed by rods with average width of $15 \mathrm{~nm}$ and length of $70 \mathrm{~nm}$. (b) Absorption spectra of the AuNRs. The transversal plasmon resonance is located around $550 \mathrm{~nm}$ and the longitudinal plasmon resonance around $920 \mathrm{~nm}$. Note, that the absorption features of the SWNTs are strongly cloaked by the plasmonic bands of the gold nanoparticles. (c) Comparison between the absorption spectra of pristine SWNTs and $\pi$-hybrids in the $\mathrm{E}_{22}$ region. (d) Comparison between the absorption spectra of pristine SWNTs and $\pi$-hybrids in the $\mathrm{E}_{11}$ region. The $\pi$-hybrid spectra in (c) and (d) have been acquired using an AuNRs blank solution. 
Figures 2c,d display the SWNTs absorption spectra in the pristine (black, continuous) and $\pi$ hybrids (blue, dashed) samples. Please note that the SWNTs' absorption bands are at least one order of magnitude weaker than the plasmon ones. With standard experimental routines, no difference would be detectable between the absorption spectra of the $\pi$-hybrids and the one of the AuNRs. To evaluate the contribution of the SWNTs in the absorption spectra of the $\pi$ hybrids, we exploited the fact that our spectrophotometer has the two separate beams, one for the blank and the other for the sample channel. We thus placed a cuvette with the AuNRs along the blank beam line and a cuvette with the $\pi$-hybrids along the sample line. The comparison between the two beams provides the SWNTs absorption bands. The intensity of the two beams can be independently adjusted and compensated to take in account differences in the optical densities of the blank and sample channels. This fine-tuning in the comparison between the two channels let us measure the relative contribution of the nanotubes in the absorption spectrum of the hybrids. The absorption spectra of pristine tubes have been acquired following the standard procedure, placing a cuvette filled with water along the blank channel. After hybridization, we observe a decrease of the band associated with smalldiameter tubes and an increase of the one associated with bigger diameter tubes. The fullwidth at half maximum of all the bands increased as well.

We moreover conducted $2 d$ excitation-emission measurements to identify the tubes present in our samples [1] and their hybridization-induced enhancement factors. Figure 3a,b show the PLE maps. Each spot represents an individual SWNT species. The luminescence enhancement factors (reported in Table 1) range between 1.3 and 4.8. The strongest enhancement occurs for the nanotubes species whose excitation window matches the TP resonance of the AuNRs, in particular the $(7,6)$ and the $(8,4)$ species. Interestingly, the highest enhancement has been observed for nanotube species red-shifted with respect to the absorption maximum. This is consistent with similar observations in simple molecular 

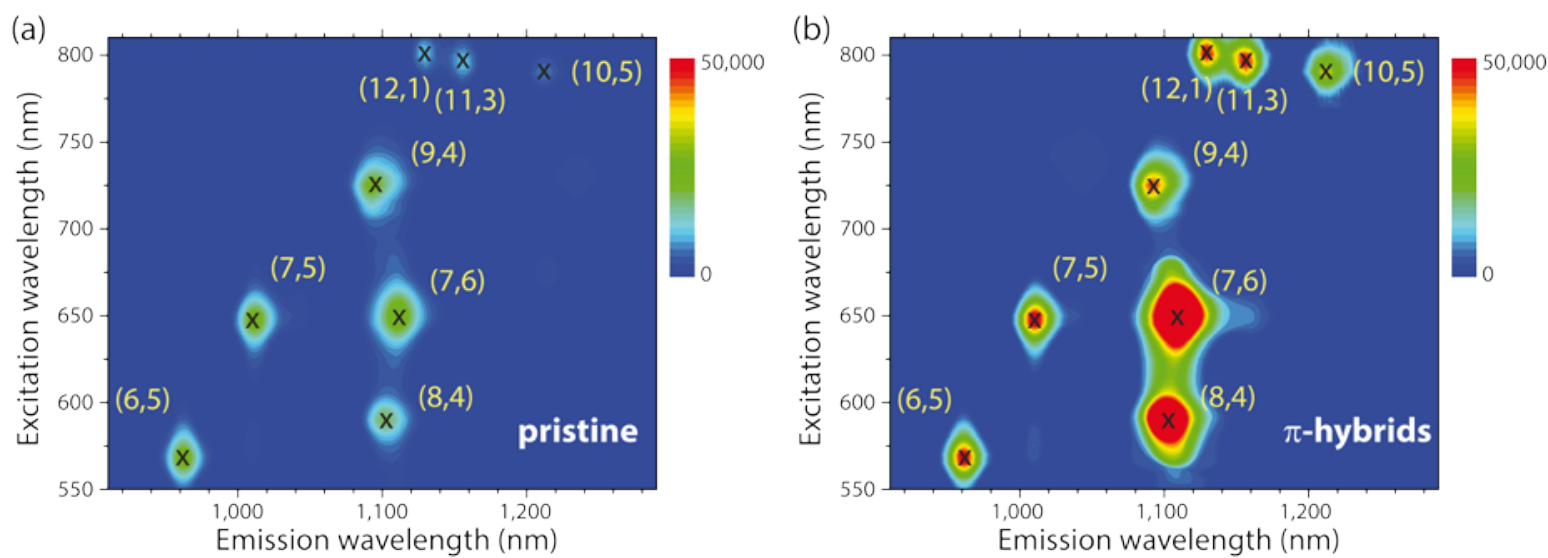

Figure $32 d$ excitation-emission map of pristine (a) and $\pi$-hybrids (b) samples. The enhancement factors are summarized in Table 1.

systems [21] and in covalently functionalized carbon nanotubes [22]. Please note that, while the TP resonance of our AuNRs occur in the excitation window of some of our SWNT species, the LP modes, with their longer wavelengths, are out of the excitation window of our SWNTs and do not contribute to the enhancement process. As mentioned before, we need to monitor the behavior of nanotubes outside the plasmon resonance window to ensure the detuning condition. The most suitable candidates for this purpose are the tubes belonging to the laola ${ }^{1}$ family with index $q=25$ (i.e. the $(12,1),(11,3)$, and $(10,5)$, cfr. Fig. 3a), which satisfy the plasmon detuning condition and match resonantly with the wavelength of our pump laser. In particular, we monitored the response of the single $(12,1)$ species, whose emission lies within the spectral region of our probe beam.

Figures 3 compare the emission intensities before and after hybridization. The resonance window of the nanotubes does not spectrally shift after hybridization: Changes in intensity thus directly relate to changes of the emission yield of the $\pi$-hybrids. The enhancement factors are summarized in Table 1.

\begin{tabular}{|c|c|c|c|c|c|c|c|c|}
\hline nantube species & $(6,5)$ & $(8,4)$ & $(7,5)$ & $(7,6)$ & $(9,4)$ & $(12,1)$ & $(11,3)$ & $(10,5)$ \\
\hline enhancement factor & 1.3 & 4.4 & 2.3 & 4.8 & 2.3 & 2.2 & 1.9 & 2.4 \\
\hline
\end{tabular}

Table 1: Enhancement factors of the different nanotube species present within our samples.

\footnotetext{
${ }^{1}$ A laola family is a set of nanotubes species sharing the same family index $q=2 n_{1}+n_{2}$.
} 


\subsection{Time-Resolved Spectroscopy}

Upon optical excitation, SWNTs undergo a de-excitation process with a fast and a slow component [23],[24]. The fast part takes place on a timescale of hundreds of femtoseconds up to one picosecond whereas the slow part develops over a scale of few tens of picoseconds. Such a double-exponential trend is observed in amorphous carbon nanoparticles as well, where the involved time constants are greater by one order of magnitude [25]. In SWNTs, the fast component is ascribed to the relaxation processes that thermalize the system at the bottom of the first excited state. This process is nonradiative. Its equivalent in the simplified Jablonski diagram of Fig. $1 \mathrm{~b}$ is the transition from $\mathrm{E}_{22}$ to the bottom of $\mathrm{E}_{11}$. The slow component, on the other hand, corresponds to the transition from the bottom of $E_{11}$ to the ground state. This part can include radiative and non-radiative de-excitation pathways. To monitor the temporal response of an excited system, one can resort to either pump-probe transient-absorption or to time-resolved photoluminescence spectroscopy. It is worth pointing out that the two techniques are inherently different, as they monitor different physical quantities: Time-resolved photoluminescence spectroscopy monitors the population change in the emissive state and provides an estimation of the radiative lifetime. Pump-probe transient absorption spectroscopy, on the contrary, follows the system de-excitation through all pathways, radiative and non-radiative, determining the total lifetime of a certain level. In our case, transient absorption monitors the whole process, starting from the $E_{22}$-to- $E_{11}$ thermalization (the fast, nonradiative process) up to the de-excitation from the $E_{11}$ to the ground state (the slow process, through radiative and non-radiative channels). Time-resolved photoluminescence, on the contrary, monitors the only radiative events that start from $\mathrm{E}_{11}$. Please note that also in time-resolved photoluminescence experiments double-exponential behaviors are observed but occurring on bigger time-scales than the phenomena in transient absorption. Their origin requires considering the excitonic response of the nanotubes 
[20],[26],[27]. As a matter of fact, the low-dimensionality and efficient suppression of dielectric screening makes the excitonic binding energies in SWNTs remarkably high (hundreds of meV and more) [28],[29], so that their optical spectra are ruled by excitons [30]. The double-exponential trend observed in time-resolved experiments is due to the balance between dark and bright excitonic states [20],[26],[27]. Thus, the decay times estimated by the two techniques are not directly comparable.

In this manuscript, we monitor the total lifetime of $\mathrm{E}_{11}$ and its changes arising from the additional de-excitation pathways (radiative or non-radiative) due to the metal presence. The most appropriate technique is thus transient absorption. For consistency sake with the most recent investigations by two-color pump and probe of the carbon nanotubes response [24],[31] we adopt the notation by Dyatlova et al. when referring to fast (intra-band) and slow (interband) components of the decay. We emphasize that those are not the fast and slow components observed in time-resolved photoluminescence experiments, which are instead due to the balance between bright and dark excitonic states and occur on longer time scales than the ones monitored here. The slow component of our transient absorption spectra $\tau_{\text {slow }}$ carries the information about the total lifetime through all, radiative and nonradiative, de-excitation channels and corresponds to $\tau_{0}$ in Eq. 3. This slow component changes when interacting with gold, giving $\tau_{\mathrm{m}}$ in Eq. 3 .

The investigation needs to be performed in the detuned configuration, avoiding overlap between the tubes and gold energy bands to get rid of spurious effects [32]. The detuning configuration is warranted because, at resonance with the plasmon frequencies, the strong local enhancement of the electromagnetic field could alter the energetic structure of tubes, for example resulting in hybridized plasmon-exciton states or even more complex phenomena. To this purpose, among the different possible nanotube species, each described by its characteristic chiral vector $\left(n_{1}, n_{2}\right)$ and its unique values of the band-gap [1], it is important to 
select a species lying outside the plasmonic window of the metallic particles. We monitor the response of the $(12,1)$ nanotube, which satisfies the de-tuning requirement and can be resonantly excited with the fundamental beam of our pump laser (Ti:Sa laser, $800 \mathrm{~nm}$ ). Please note that that altering the size of the metallic nanoparticles would cause a shift of the spectral position of the plasmonic bands and break the detuning condition as well.

We monitored the dynamics of our system with a two-color pump-probe system, according to the configuration established by Dyatlova et al. [24]. The de-excitation dynamics of our samples follow a double-exponential behavior, exhibiting a fast, sub-picosecond component ( $\tau_{\text {fast }}<1$ ps) and a slow component of several picoseconds $\left(\tau_{\text {slow }}>10 \mathrm{ps}\right.$ ), see Fig. 4a. The obtained lifetimes are consistent with the values previously reported in literature [20],[24]. The lifetimes of our $\pi$-hybrids are shorter than the ones of pristine nanotubes, see Fig. $4 \mathrm{~b}$-d. This is consistent with experimental observations in analogous but simpler nanometal-emitter hybrid systems, e.g. dyes attached to gold nanoparticles, where the dye lifetime shortens after interacting with the metal [33].

However, nanotubes are more complex than simple fluorophores. They exhibit a fast component, during which the system undergoes a nonradiative thermalization, and a slow component, during which radiative and non-radiative interband recombination occurs. Both regimes can easily be identified in Fig. 4a.

The trend of the fast and slow time constants for pristine samples and $\pi$-hybrids are reported in Fig. 4c,d. $\tau_{\text {fast }}$ goes from (370 \pm 20$)$ fs for pristine SWNTs to $(180 \pm 40)$ fs for the $\pi$-hybrids. Similarly, $\tau_{\text {slow }}$ goes from $(16 \pm 3)$ ps for the pure sample to $(12 \pm 5)$ ps for the $\pi$-hybrids. After hybridization, the fast decay component changes $\left(\frac{\Delta \tau_{\text {fast }}}{\tau_{\text {fast }}}=106 \%\right)$ much more acutely than the slow component $\left(\frac{\Delta \tau_{\text {slow }}}{\tau_{\text {slow }}}=33 \%\right)$.

In Fig. 4b we compare the first few picoseconds of the de-excitation process to highlight the 

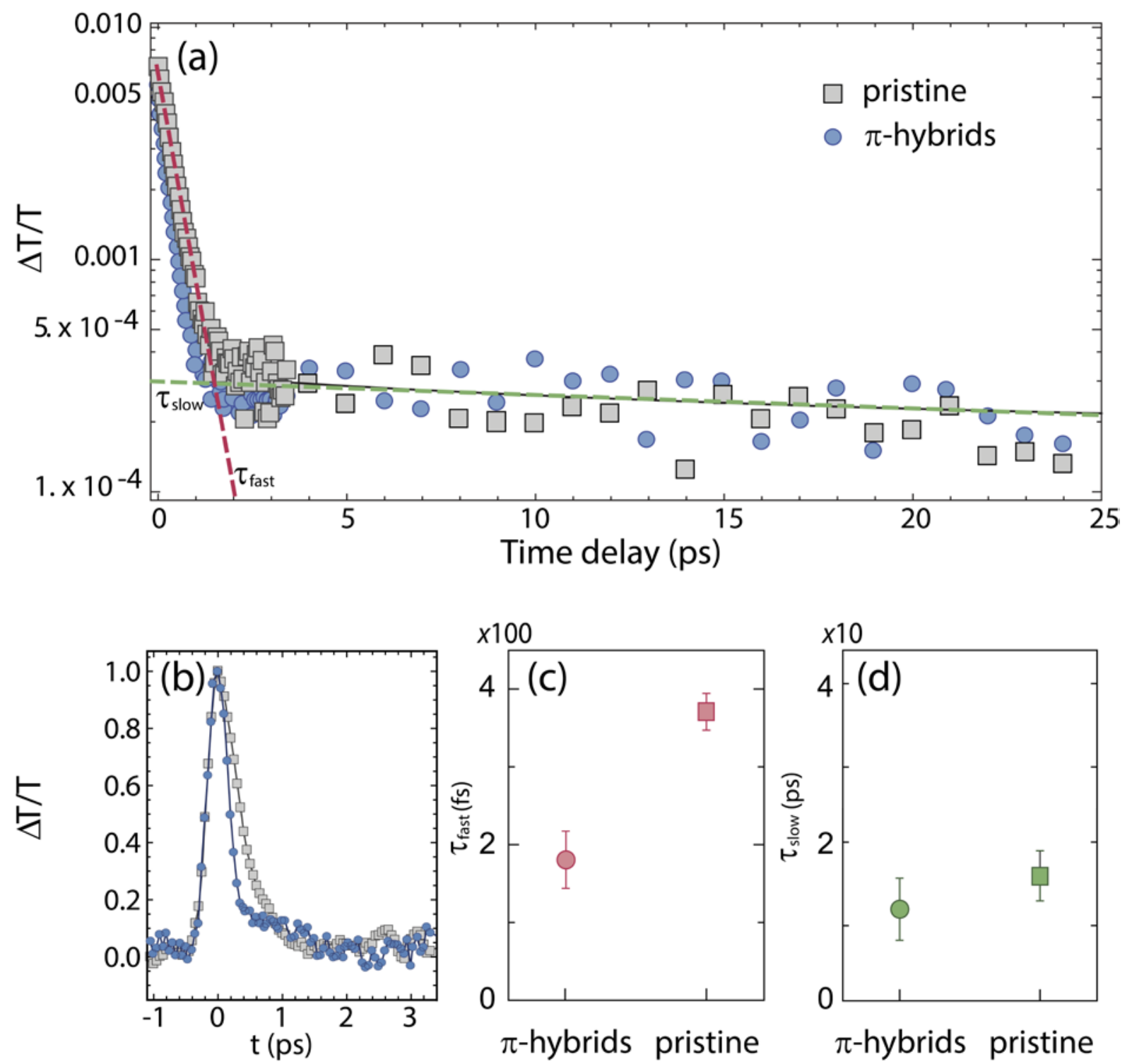

Figure 4 (a) Time-resolved response of the (12,1) SWNT species of the $\pi$-hybrids (blue) vs. pristine nanotubes (gray). (b) Comparison of the fast de-excitation process of the $\pi$-hybrids (blue) vs. pristine nanotubes (gray). (c) Comparison between the time constant of the fast process for the $\pi$-hybrids (circle) and pristine tubes (squares). (d) Comparison between the time constant of the slow process for the $\pi$-hybrids (circles) and pristine tubes (squares).

speeding up of the hybrid fast-process. Our experimental results clearly show that the presence of gold nanoparticles shortens the slow and, even more acutely, the fast relaxation times of the SWNTs. Note that no signal can be detected from the transient response of a pure AuNRs suspension within our pump window. The response is either too weak or too fast to be disentangled from the IRF.

These observations are consistent with the overall trend observed previously where the 
relaxation times were found to be slower in isolated SWNTs vs. SWNT bundles or SWNTpolymer mixtures, indicating that tube-tube or tube-environment interactions provide additional decay channels [34],[35]. While the presence of gold nanoparticles is expected to locally alter the dielectric environment around the SWNTs, thus altering both their electronic [36] and phonon band structure [37],[38], the exact mechanisms behind the influence of the gold nanoparticles on the SWNTs await elucidation via further experimental and theoretical work.

Obtaining an estimation of the decay times lets us evaluate the contribution of the two terms of the quantum yield of our system, allowing us, for the first time in such systems, to disentangle whether the enhancement is mainly due to stronger pumping or faster deexcitation (Eq. 1-3). By inserting the experimental time constants into Eq. 3, we obtain that the lifetime-change term contributes to the enhancement with a value $F_{\text {rad }}=(0.7 \pm 0.3)$. This implies that the local-field contribution is $F_{\text {exc }}=(3 \pm 1)$. Note that our estimate of $F_{\text {rad }}$ might be biased by the neglect of Joule losses that are candidate nonradiative decay channels in plasmonic systems [39].

The stronger emission from our $\pi$-hybrids is not governed by the faster de-excitation, as previously observed for SWNTs dropcasted onto rough Au surfaces [6]. We instead find that the stronger pumping due to the localized plasmonic near-field actually drives the enhancement, compensating the nonradiative losses from the metal. We believe that this due to the homogeneous exposure of the SWNTs to the plasmonic near-field ensured by the micelle encapsulation, rather than the less-controllable match with the plasmonic hot-spots of the rough metallic surfaces. This finding is highly relevant for the improvement of the enhancement process, indicating that the parameter to tailor for improving the efficiency of the $\pi$-hybrids' enhancement process is the intensity of the near-field localized around the surface of the metallic particles. 


\section{Conclusion}

We performed two-color pump-probe measurements to investigate the decay dynamics of carbon nanotubes in the close vicinity of gold nanoparticles. We observe an overall speeding up of the de-excitation dynamics due to the metal proximity. The emission-enhancement of our $\pi$-hybrids is mainly driven by the stronger pumping through the localized plasmonic nearfield rather than by the additional de-excitation pathways due to the metal presence. These findings provide a guide for efficient fabrication of further enhanced $\pi$-hybrids and stimulate further studies of the microscopic mechanisms behind the near-field interactions. To fully exploit the enhancement potential of the SWNTs, we will resort to a rational design of tailored metallic nanoparticles with increased intensity of the near fields localized around them. Structures with more singular morphologies -such as pyramids or nanostars- are promising candidates in this regard. 


\section{Aknowledgment}

M.G. and A.S. gratefully thank the FU Focus Area NanoScale for financial support. Part of this work also has been granted by the SFB 658, subproject A6. We further would like to thank S. Reich for her valuable support. A.B. wishes to thank the project Laboratorio Congiunto "Amorphous materials for energy harvesting applications" funded by Consiglio Nazionale delle Ricerche, for partial financial support.

\section{References}

[1] O'Connell MJ; Bachilo SM; Huffman CB; Moore VC; Strano MS; Haroz EH; Rialon KL; Boul PJ; Noon WH; Kittrell C; Ma J; Hauge RH; Weisman RB; Smalley RE. 2002 Band gap fluorescence from individual single-walled carbon nanotubes Science 297 593-596

[2] Hagen A; Steiner M; Raschke MB. Lienau C; Hertel T; Qian H; Meixner AJ; Hartschuh A. 2005 Exponential Decay Lifetimes of Excitons in Individual Single-Walled Carbon Nanotubes Phys. Rev. Lett. 95197401

[3] Tsyboulski DA; Rocha JDR.; Bachilo SM; Cognet L; Weisman RB. 2007 StructureDependent Fluorescence Efficiencies of Individual Single-Walled Carbon Nanotubes Nano Lett, 7 3080-3085

[4] Reich S; Thomsen C; Maultzsch J. 2004 Carbon Nanotubes: Basic Concepts and Physical Properties (Wiley-VCH Verlag, Weinheim)

[5] Glaeske M; Setaro A. 2013 Nanoplasmonic colloidal suspensions for the enhancement of the luminescent emission from single-walled carbon nanotubes Nano Res. 6 593-601

[6] Hong G; Tabakman SM; Welsher K; Wang H; Wang X; Dai H. 2010 Metal-Enhanced Fluorescence of Carbon Nanotubes J. Am. Chem. Soc. 132 15920-15923

[7] Zakharko Y; Graf A; Zaumseil J. 2016 Plasmonic Crystals for Strong Light-Matter Coupling in Carbon Nanotubes. Nano Lett. 16 6504-6510

[8] Graf A; Tropf L; Zakharko Y; Zaumseil J; Gather MC. 2016 Near-infrared excitonpolaritons in strongly coupled single-walled carbon nanotube microcavities Nat. Comm. 7 13078

[9] Kumar A; Kim S; Nam JM. 2016 Plasmonically Engineered Nanoprobes for Biomedical Applications J. Am. Chem. Soc. 138 14509-14525 
[10] Lakowicz JR. 2006 Principles of Flourescence. Springer

[11] Sakashita T; Miyauchi Y; Matsuda K; Kanemitsu Y. 2010 Plasmon-assisted photoluminescence enhancement of single-walled carbon nanotubes on metal surfaces Appl. Phys. Lett. 97063110.

[12] Sun G; Khurgin JB; Soref RA. 2009 Practical enhancement of photoluminescence by metal nanoparticles Appl. Phys. Lett. 94101103

[13] Kern AK; Zhang D; Brecht M; Chizhik AI; Failla AV; Wackenhuta F;. Meixner AJ. 2014 Enhanced single-molecule spectroscopy in highly confined optical fields: from $\lambda / 2$ Fabry-Pérot resonators to plasmonic nano-antennas Chem. Soc. Rev. 431263

[14] Glaeske M; Setaro A. 2014 Effect of hybrid isolation on the luminescence enhancement of carbon nanotube-gold nanorod composites Phys. Status Solidi B. 251 2480-2484

[15] Ye X; Zheng C; Chen J; Gao Y; Murray C. 2013 Using Binary Surfactant Mixtures To Simultaneously Improve the Dimensional Tunability and Monodispersity in the Seeded Growth of Gold Nanorod Nano Letters 13 765-771

[16] Panahandeh-Fard M; Yin J; Kurniawan M; Wang Z; Leung G; Sum TC; Soci C. 2014 Ambipolar Charge Photogeneration and Transfer at GaAs/P3HT Heterointerfaces The Journal of Physical Chemistry Letters 5 1144-1150

[17] Demas JN. Excited state life time measurements 1983 (Academic Press, New York)

[18] O’Connor DV; Phillips D. 1994 Time Correlated Single Photon Counting (Academic Press, New York)

[19] Bruno A; de Lisio C; Minutolo P. 2005 Time resolved fluorescence polarization anisotropy of carbonaceous particles produced in combustion systems Optics Express 13 5393-5408

[20] Gokus T; Hartschuh A; Harutyunyan H; Allegrini M; Hennrich F; Kappes M; Green AA; Hersam MC; Araújo PT; Jorio A. 2008 Exciton decay dynamics in individual carbon nanotubes at room temperature Appl. Phys. Lett. 92153116

[21] Anger P; Bharadwaj P; Novotny L. 2006 Enhancement and Quenching of SingleMolecule Fluorescence Phys. Rev. Lett. 96113002

[22] Setaro A; Adeli A; Glaeske M; Przyrembel D; Bisswanger T; Gordeev G; Maschietto F; Faghani A; Paulus B; Weinelt M; Arenal R; Haag R; Reich S. 2017 
Preserving $\pi$-conjugation in covalently functionalized carbon nanotubes for optoelectronic applications Nature Communications 814281

[23] Ostojic GN; Kono J. 2004 Interband Recombination Dynamics in Resonantly Excited Single-Walled Carbon Nanotubes Physical Review Letters 92117402

[24] Dyatlova OA; Koehler C; Vogel P; Malic E; Jain RM; Tvrdy KC; Strano MS; Knorr A.; Woggon U. 2014 Relaxation dynamics of carbon nanotubes of enriched chiralities Phys. Rev.B 90, 155402

[25] Bruno A; de Lisio C; Minutolo P; D’Alessio A. 2006 Detection of fluorescent nanoparticles in flame with femtosecond laser-induced fluorescence anisotropy Journ. of Optics A 8 5623-5632

[26] Duque J; Pasquali M; Cognet L; Lounis B. 2009 Environmental and SynthesisDependent Luminescence Properties of Individual Single-Walled Carbon Nanotubes ACS Nano 3 2153-2156

[27] Gokus T; Cognet L; Duque JG; Pasquali M; Hartschuh A; Lounis B. 2010 Mono- and Biexponential Luminescence Decays of Individual Single-Walled Carbon Nanotubes. J. Phys. Chem. C 114 14025-14028

[28] Maultzsch J; Pomraenke R; Reich S; Chang E; Prezzi D; Ruini A; Molinari E; Strano MS; Thomsen C; Lienau C. 2005 Exciton binding energies in carbon nanotubes from twophoton photoluminescence Phys. Rev. B 72 241402(R)

[29] Maultzsch J; Pomraenke R; Reich S; Chang E; Prezzi D; Ruini A; Molinari E; Strano MS; Thomsen C; Lienau C. 2006 Excitons in Carbon Nanotubes Phys. Stat. Sol. B 243 3204-3208

[30] Wang F; Dukovic G; Brus LE; Heinz TF. 2005 The Optical Resonances in Carbon Nanotubes Arise from Excitons Science 308 838-841

[31] Dyatlova OA;. Köhler C; Malic E; Gomis-Bresco J; Maultzsch J; TsaganMandzhiev A; Watermann T; Knorr A; Woggon U. 2012 Ultrafast Relaxation Dynamics via Acoustic Phonons in Carbon Nanotubes Nano Letters 12 2249-2253

[32] Darby BL; Auguié; Meyer BM; Pantoja AE; Le Ru EC. 2016 Modified optical absorption of molecules on metallic nanoparticles at sub-monolayer coverage Nature Photonics 10 40-45

[33] Fu Y; Zhang J; Lakowicz JR. 2010 Plasmon-Enhanced Fluorescence from Single Fluorophores End-Linked to Gold Nanorods J. Am. Chem. Soc. 132 5540-5541 
[34] Hertel T; Moos G. 2000 Electron-Phonon Interaction in Single-Wall Carbon Nanotubes: A Time-Domain Study Phys. Rev. B 84 5002-5005

[35] Zamkov M; Woody N; Shan B; Chang Z; Richard P. 2005 Lifetime of Charge Carriers in Multiwalled Nanotubes Phys. Rev. Lett. 94056803

[36] Siegel DA; Park CH; Hwang C; Deslippe J; Fedorov AV; Louie SG; Lanzara A. 2011 Many-body interactions in quasi-freestanding graphene PNAS 108 11365-11369

[37] Piscanec S; Lazzeri M; Mauri F; Ferrari AC; Robertson J. 2004 Kohn Anomalies and Electron-Phonon Interactions in Graphite Phys. Rev. Lett. 93185503

[38] Narula R; Reich S. 2014 Graphene band structure and its 2D Raman mode. Phys. Rev. B 90085407

[39] Tassin P;. Koschny T; Kafesaki M; Soukoulis CM. 2012 A comparison of graphene, superconductors and metals as conductors for metamaterials and plasmonics Nat. Photonics 6 259-264 